\title{
Nonmagnetic Insulating States near the Mott Transitions on Lattices with Geometrical Frustration and Implications for $\kappa-(\mathrm{ET})_{2} \mathrm{Cu}_{2}(\mathrm{CN})_{3}$
}

\author{
Hidekazu Morita, Shinji Watanabe and Masatoshi Imada \\ Institute for Solid State Physics, University of Tokyo, \\ Kashiwanoha, Kashiwa, Chiba, 277-8581, Japan
}

\begin{abstract}
We study phase diagrams of the Hubbard model on anisotropic triangular lattices, which also represents a model for $\kappa$-type BEDT-TTF compounds. In contrast with mean-field predictions, path-integral renormalization group calculations show a universal presence of nonmagnetic insulator sandwitched by antiferromagnetic insulator and paramagnetic metals. The nonmagnetic phase does not show a simple translational symmetry breakings such as flux phases, implying a genuine Mott insulator. We discuss possible relevance on the nonmagnetic insulating phase found in $\kappa-(\mathrm{ET})_{2} \mathrm{Cu}_{2}(\mathrm{CN})_{3}$.
\end{abstract}

When the kinetic and Coulomb repulsion energies are severely competing, the ground states of many-body electron systems can be highly nontrivial. The Mott transition provides a typical example of such nontrivial behavior. If the electronic orbitals constituting the band near the Fermi level form geometrically frustrated lattice structure, the ground state can be even more nontrivial, because the spin entropy is not easily released by simple symmetry breaking such as the antiferromagnetic (AF) order, and the possiblity of some exotic quantum liquid state opens. Theoretically, regular triangular lattice has been proposed as such a typical structure [1], while the nature of the ground state of the Hubbard model on this lattice still remains a challenge because of the lack of theoretical tools. Reliability of mean field approaches becomes questionable under strong quantum fluctuations while the numerical studies have been limited to small clusters because of the lack of efficient algorithm.

In this letter, we obtain the ground-state phase diagram of the Hubbard model on anisotropic triangular lattice called $t-t^{\prime}-\mathrm{TH}$ model by using recently developed path-integral renormalization group (PIRG) method [2] with systematic inclusion of quantum fluctuations. In the $t-t^{\prime}-\mathrm{TH}$ model, when one of the transfers $\left(t^{\prime}\right)$ on a unit triangle becomes different from the other transfers $t$ as in Fig. 1, the model continuously connects the right triangular lattice $\left(t^{\prime} / t=1\right)$ to the regular square one $\left(t^{\prime} / t=0\right)$. We study this general model at half filling.

In particular, the case between $t^{\prime} / t \sim 0.5$ and 1.0 has attracted a particular interest because tight binding fit of the band structure by the extended Hückel approximation for $\kappa$-type BEDT-TTF compound suggests that this region may provide a minimal model after eliminating

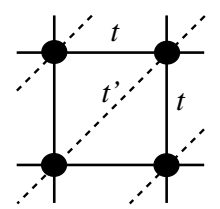

FIG. 1: Lattice structure of tt'-TH model on triangular lattice with anisotropic transfers $t$ and $t^{\prime}$. the internal degrees of freedom in the dimerized two ET molecules [3]. In terms of clarifying the metal-insulator (MI) transition and the accompanied superconductivity, the ground state of this model has been a subject of intensive studies but mainly on the Hartree-Fock or FLEX levels 田, 5, 6, 7]. Because significant correlation and quantum effects are expected as described above, it is desired to clarify the phase diagram of the basic $t-t^{\prime}-\mathrm{TH}$ models itself by fully taking account of quantum fluctuations.

Our result shows remarkably a generic emergence of nonmagnetic insulating state sandwitched by a Mott MI transition and an AF transition in contrast with the previous mean-field predictions, while a nonmagnetic insulating state is also suggested in different Hubbard models on the square lattice with next-nearest neighbor transfers [8]. We also find that the nonmagnetic insulators do not show simple symmetry breakings such as dimer, plaquette, and flux phases, in support for the appearance of genuine Mott insulator distinct from band insulators.

The genuine Mott insulating state not adiabatically connected to the band insulator has been a subject of long-standing search since Anderson's proposal [1]. This proposed RVB state is in basic sense realized in the 1D systems, while it remains a challenge for multidimensional systems. We stress that such RVB state may be found more easily near the bandwidth-control metal to Mott insulator transition, where quantum mechanical fluctuations of spin as well as charge destroy trivial symmetry breakings and the local moment is relatively small. In the light of our results, we argue that this category of genuine Mott insulator may indeed be realized in $\kappa$ - $(\mathrm{ET})_{2} \mathrm{Cu}_{2}(\mathrm{CN})_{3}$, which seems to be nonmagnetic and insulating at ambient pressure down to $1.4 \mathrm{~K}$.

We introduce $t$ - $t^{\prime}$-TH model on a triangular lattice shown in Fig. 1 with the Hubbard Hamiltonian $H=$ $-\sum_{\langle i, j\rangle, \sigma} t\left(c_{i \sigma}^{\dagger} c_{j \sigma}+\right.$ H.c. $)+\sum_{\langle k, l\rangle, \sigma} t^{\prime}\left(c_{k \sigma}^{\dagger} c_{l \sigma}+\right.$ H.c. $)+$ $U \sum_{i=1}^{N} n_{i \uparrow} n_{i \downarrow}$ with the standard notation. In this letter, we apply the recently developed PIRG method [2] to clarify the ground state in the plane of $U / t$ and $t^{\prime} / t$. This algorithm allows us to start from and improve a mean-field Hartree-Fock solution and reach the ground-state by taking account quantum fluctuations in a systematic fashion. 


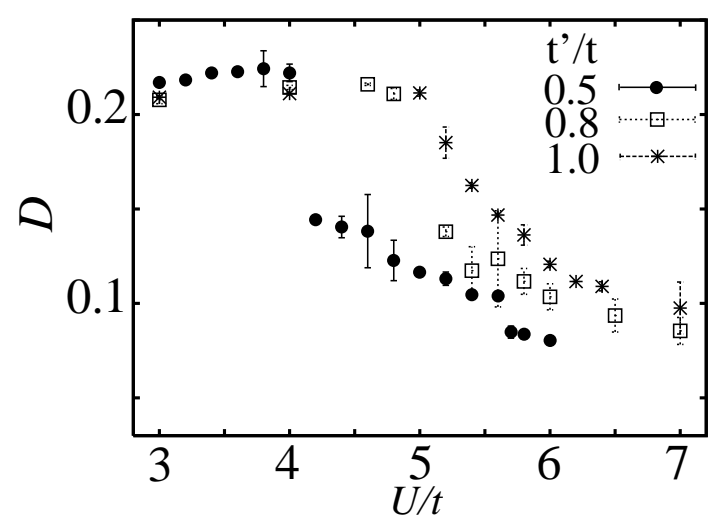

FIG. 2: Averaged double occupancy $D$ as a function of $U / t$ for $t^{\prime} / t=0.5$ (filled circle), 0.8(open square) and 1.0(asterisk). The plotted error bars are only from the size extrapolation. The errors produced by the variance extrapolation[2] in PIRG is not shown and are at most 0.02 .

By increasing the dimension of truncated Hilbert space in a nonorthogonal basis numerically optimized by the path-integral operation, a variance extrapolation is taken to reach the true ground state of finite size systems in a controlled way. We took number of Slater basis functions up to around 300. This numerical method does not have difficulties such as the negative sign problem in the quantum Monte Carlo calculations. In the present study, we take system size up to $N=196$ sites $(14 \times 14)$ with the periodic boundary condition. Using the results at various sizes, we extrapolate to the thermodynamic limit using appropriate scaling procedure and eliminate finite size effects.

To see the MI transition, we calculate the singularity of the ground-state energy $E_{g}$ as a function of the control parameter, $U / t$. The level crossing with a first-order transition or singularity with a continuous character is detected as a signature of the MI transition with complementary analysis of the charge gap and the momentum distribution. The AF transition is studied by equal-time spin correlation functions in the momentum space. We also calculate several density wave correlations to discuss possible symmetry breakings such as staggered flux as well as dimer correlations.

In Fig. 2, we show $\partial E_{g} / \partial U$ as a function of $U$. Jumps of the averaged double occupancy $D=\left\langle n_{i \uparrow} n_{i \downarrow}\right\rangle=$ $\partial E_{g} / \partial U / N+1 / 4$ signal first-order MI transitions induced by level crossings at $U / t=4.1 \pm 0.1$, and $5.0 \pm 0.2$ for choices $t^{\prime} / t=0.5$, and 0.8 , respectively, while a continuous transition is plausible at $5.2 \pm 0.2$ for $t^{\prime} / t=1.0$. These transitions show similar character to MI transitions identified by charge gap formation and qualitative change of the momentum distributions for other types of Hubbard models [8]. Fig. 3 exemplifies that the momentum distributions of the $t-t^{\prime}$-TH model indeed show transition from a typical metallic behavior to that of an insulator across this boundary .

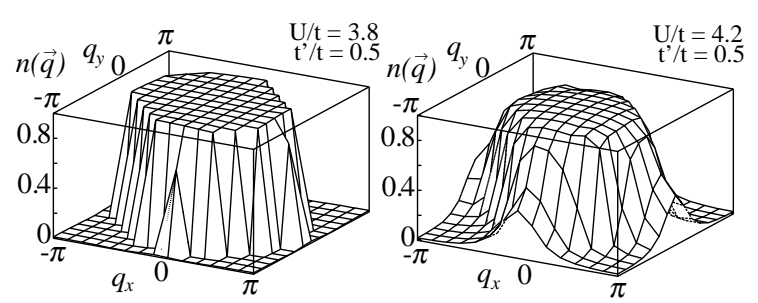

FIG. 3: Momentum distribution on $14 \times 14$ lattice at $t^{\prime} / t=$ 0.5 for $U / t=3.8$ and 4.2 .

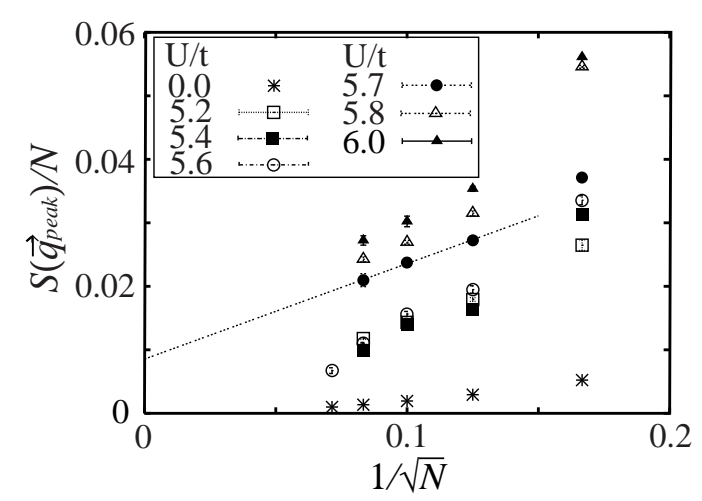

FIG. 4: Size scaling for the peak value of the equal-time spin structure factor $S\left(q_{\text {peak }}\right)$ at $t^{\prime} / t=0.5$.

The magnetic transition is probed by the Fourier transform of the equal-time spin correlation, $S(\boldsymbol{q})$ defined by $S(\boldsymbol{q})=\frac{1}{3 N} \sum_{i, j}^{N}\left\langle\boldsymbol{S}_{i} \cdot \boldsymbol{S}_{j}\right\rangle e^{i \boldsymbol{q} \cdot\left(\boldsymbol{R}_{i}-\boldsymbol{R}_{j}\right)}$, where $\boldsymbol{S}_{i}$ is the spin of the $i$-th site with $\boldsymbol{R}_{i}$ representing the coordinate of the $i$-th site. At $t^{\prime} / t=0.5$ the spin correlation shows sharp commensurate paek at $(\pi, \pi)$ for $U / t \geq 5.7$ while it remains rather small and incommensurate for $U / t \leq 5.6$. The size scaling in Fig. 1 leads to $U$ dependence of the staggered moment $m=\sqrt{\lim _{N \rightarrow \infty} S\left(\boldsymbol{q}_{\text {peak }}\right) / N}$ as in Fig. 5, where a first-order (or very sharp continuous) AF transition is identified at $U / t=5.65 \pm 0.05$. A small jump of $\partial E_{g} / \partial U$ seen in Fig. 2 at this point also supports the first-order character. For larger values of $t^{\prime} / t$, at $t^{\prime} / t=0.8$ and 1.0 , the size scaling of the spin correlation shows the absence of magnetic symmetry breaking at all momenta and all $U$ studied, namely for $U / t \leq 10$.

The phase diagram of the $t-t^{\prime}$-TH model constructed from our calculations is illustrated in Fig. 6. A remarkable feature is the emergence of nonmagnetic insulating phase sandwitched by MI and AF transitions. This is in contrast with the Hartree-Fock results where the MI transition occurs at larger $U / t$ than the AF transition. The triangular lattices were also studied in the spin- $1 / 2$ Heisenberg model corresponding to the limit $U \rightarrow \infty$ [9, 10]. Although the decisive conclusion cannot 


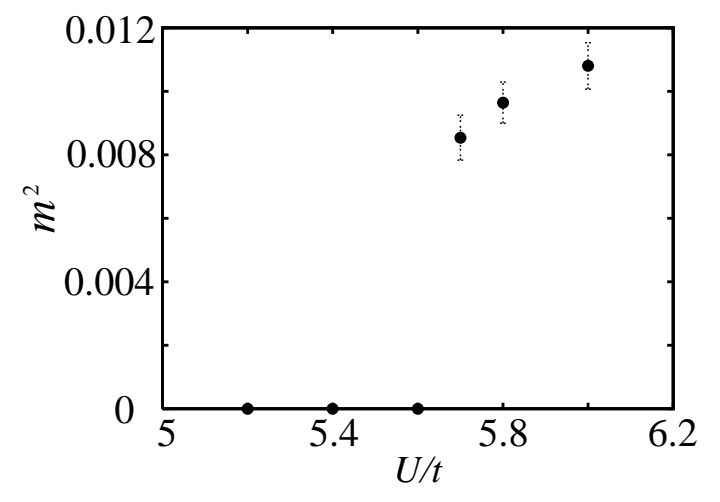

FIG. 5: Staggered magnetization in the thermodynamic limit as a function of $U / t$ at $t^{\prime} / t=0.5$.

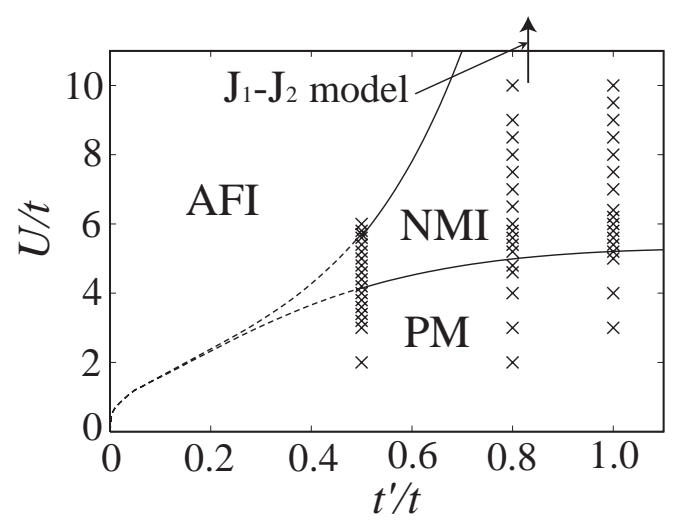

FIG. 6: Phase diagram of $t-t^{\prime}$ TH model in the plane of $U / t$ at $t^{\prime} / t$. AFI,NMI and PM represent AF insulator, nonmagnetic insulator, and paramagnetic metal, respectively. Calculations have been done at the cross points. The arrow shows speculated boundary from AFI to the dimer insulator in the limit $U \rightarrow \infty$ for the $J_{1-} J_{2}$ model [10].

be drawn due to the lack of powerful techniques, it was claimed from a small cluster studies or series expansions that either long-range AF order or dimer order appear in this model on a regular triangular lattice. This suggests a possibility that the reduction of the local moment due to charge fluctuations at $U$ reduced to a finite value drives the destruction of the order. In fact, for example, the local moment $m_{l}=\sqrt{\langle n\rangle-2 D} / 2$ is reduced to $m_{l} \sim 0.4$ at the phase boundary $U / t=5.2$ at $t^{\prime} / t=1.0$.

Insulators with some translational symmetry breakings such as the AF and dimer orders may be adiabatically continued to band insulators due to the band gap formation through extension of the unit cell and the folded Brillouin zone, and hence do not belong to the genuine Mott insulator discussed above. We first have to note that it is hard to prove the existence of the genuine Mott insulator because it is difficult to prove the absence of all the possible translational symmetry breakings. Un-

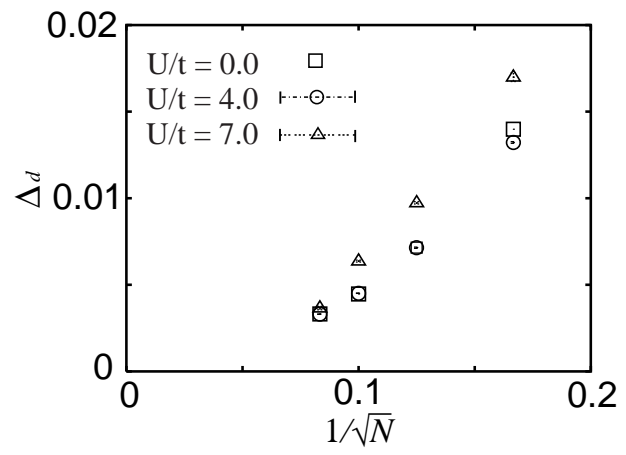

FIG. 7: Size scaling for the d density wave (stuggered flux) correlation $\Delta_{d}=\left|D_{1 d}\left(\boldsymbol{q}_{\text {peak }}\right)\right| / N$ for $t^{\prime} / t=1.0$.

der this circumstance, an available way to get insight on this problem is to examine every symmetry breaking ever proposed and judge the plausibility of such a phase.

Along this line of motivation, we have further calculated correlations for several symmetry breakings claimed in the literature such as dimer order [10, 11], plaquette order 12] and various density waves [13] in this remarkable nonmagnetic insulating phase. Here the density wave correlation is defined by $D_{\alpha}(\boldsymbol{q})=\left\langle\Delta_{\alpha}^{\dagger}(\boldsymbol{q}) \Delta_{\alpha}(\boldsymbol{q})\right\rangle$ where $\Delta_{\alpha}^{\dagger}(\boldsymbol{q})=\sum_{\boldsymbol{k}, \sigma} f_{\alpha}(\boldsymbol{k}) c_{\boldsymbol{k}_{\sigma}}^{\dagger} c_{\boldsymbol{k}+\boldsymbol{q}, \sigma}$. We have studied the cases $f_{1 s}(\boldsymbol{k})=\cos k_{x}+\cos k_{y}, f_{2 s}(\boldsymbol{k})=2 \cos k_{x} \cos k_{y}$, $f_{1 d}(\boldsymbol{k})=\cos k_{x}-\cos k_{y}$, and $f_{2 d}(\boldsymbol{k})=2 \sin k_{x} \sin k_{y}$. We note the case $1 d$ represents the order parameter for the staggered flux (or $d$-density wave) state 13]. Fig. 7 i shows a typical size scalings of these density wave correlations for the $t-t^{\prime}-\mathrm{TH}$ model. Other types show similar behaviors. It does not seem to show symmetry breakings and the correlation actually remains rather short ranged in all the parameter space we have studied $(0 \leq U / t \leq 10$ and $\left.0.5 \leq t^{\prime} / t \leq 1.0\right)$. The possibility of dimer, plaquette and density wave orders have also been examined in the Hubbard model on a square lattice with diagonally crossing transfers between next-nearest neighbor sites. The size scalings again suggest the absence of these translational symmetry breakings [14]. Although these results do not exclude a possiblity for the translational symmetry breakings other than those studied here, we conclude that the theoretically proposed phases so far are unlikely to be realized in our phase hence the existence of genuine Mott insulating state becomes much more plausible.

Our result leads us to an intriguing view for recent experimental results on organic compounds. As we discussed above, the $t-t^{\prime}$-TH model provides a simplest effective Hamiltonian for the $\kappa$-type ET compounds if we may take the dimerization of two parallel ET molecules large [3]. The HOMO band is split by the dimerization and the upper band provides the effective Hubbard model at half filling. The effective interaction for $U$ in this case is taken as $U_{\text {eff }}=2 t_{b 1}+\frac{U_{E T}}{2}\left[1-\sqrt{1+\left(\frac{4 t_{b 1}}{U_{E T}}\right)^{2}}\right]$, where $U_{E T}$ is the original onsite Coulomb repulsion for the ET 
molecule and $t_{b 1}$ is the intradimer transfer.

Among available compounds, the band structure calculation 15, 16] suggests $\kappa$ - $(\mathrm{ET})_{2} \mathrm{Cu}_{2}(\mathrm{CN})_{3}$ has the largest ratio of $t^{\prime} / t \sim 1.0\left(t \sim 0.055 \mathrm{eV}\right.$ and $t^{\prime} \sim 0.058 \mathrm{eV}$ ) with rather large $U_{\text {eff }} / t \sim 8.2$. This compound is close to the metal-insulator phase boundary because a small pressure around $0.05 \mathrm{GPa}$ drives the insulating to the superconducting phase. The ESR measurement of $\kappa$ $(\mathrm{ET})_{2} \mathrm{Cu}_{2}(\mathrm{CN})_{3}$ at ambient pressure as well as the susceptibility seems to suggest that the insulating phase remains nonmagnetic with broad structure of the spin susceptibility [16]. Very recent NMR data [18] also suggest that the $1 / T_{1}$ remains constant above $100 \mathrm{~K}$ while it starts substantially decreasing below $50 \mathrm{~K}$ and there exists no signature of the magnetic transition down to $1.4 \mathrm{~K}$ so far. No indication of lattice distortion has been reported.

It is very attractive to relate these experimental results to our calculated phase diagram with the insulating phase without symmetry breakings, because the parameter values deduced from the band structure calculation correspond to $t^{\prime} / t=1.0$ where we obtain large nonmagnetic insulating phase. In other compounds such as deuterated $\kappa-(\mathrm{ET})_{2} \mathrm{Cu}\left[\mathrm{N}(\mathrm{CN})_{2}\right] \mathrm{Br}$, the first-order transition between a superconductor and an $\mathrm{AF}$ insulator is observed. These compounds have relatively small parameters $t^{\prime} / t \sim 0.5-0.6$ 17. In our result at $t^{\prime} / t=0.5$, there still exists nonmagnetic insulating phase. This discrepancy is speculated to be due to an oversimplification to the effective single-band model. In fact, internal degrees of freedom of dimer stabilizes metals more than insulator, while the geometrical frustration is relaxed by an assymetric distribution of electrons on a dimer and the $\mathrm{AF}$ order may not be suppressed strongly, which results in shrink of the nonmagnetic insulator phase. Necessity of multi-band models was also pointed out in different contexts 19, 20]. Quantitative analysis of this multi-band effect is left for further studies. If the AF and MI transitions become close, lattice distortion accompanied by the MI transition necessarily enhance the first-order nature also leading to the shrink of the nonmagnetic insulator.
The absence of strong AF correlations near the MI transition implies that the mechanism of the superconductivity seen in $\kappa$ - $(\mathrm{ET})_{2} \mathrm{Cu}_{2}(\mathrm{CN})_{3}$ under pressure may not be directly connected with AF fluctuations. In our PIRG calculation, although the pairing correlations definitely remain short-ranged for $U / t=3$ at $t^{\prime} / t=0.5$ and for $U / t=4$ at $t^{\prime} / t=0.8$ and 1.0 , the d-wave pairing correlations near the MI boundary does not show a good convergence which indicates the necessity of larger number of Slater determinants in PIRG for this quantity to get converged results, implying large quantum fluctuations. This will be discussed elsewhere.

We also point out, however, a fundamentally interesting region still waits for experimental realization: In ET dimerized compounds, stronger dimerization is required. More sophisticated realization of nonmagnetic insulator and metals near it in the frustrated lattice would be desired also in other materials.

In summary, we have studied the Hubbard model on anisotropic triangular lattice $\left(t-t^{\prime}-\mathrm{TH}\right.$ model). The ground state is obtained by the PIRG method, where the Hartree-Fock results are systematically improved to reach the true ground state. The phase boundary of bandwidth control MI and AF transitions in the parameter space of $t^{\prime} / t$ and $U / t$ shows that a nonmagnetic insulator appears between these two transitions. Simple translational symmetry breakings such as flux phases remain short ranged and genuine Mott insulating state seems to be realized. We have pointed out that $\kappa-(\mathrm{ET})_{2} \mathrm{Cu}_{2}(\mathrm{CN})_{3}$ may belong to this category of the genuine Mott insulator within the available data. The degeneracy of the ground state as well as the spin excitation gap in the nonmagnetic insulator phase is an intriguing future problem to be clarified.

We thank K. Kanoda for valuable discussions. The work is supported by the 'Research for the Future' program from JSPS under grant number JSPSRFTF97P01103. A part of the computation was done at the supercomputer center in ISSP, University of Tokyo.
[1] P. Fazekas and P.W. Anderson, Phil. Mag. 30423 (1974).

[2] M.Imada and T.Kashima, J. Phys. Soc. Jpn. 692723 (2000); ibid. 702287 (2001).

[3] K. Oshima et al., Phys. Rev. B 38938 (1988).

[4] R. H. Mackenzie, Comm. Cond. Mat. Phys.18 309 (1998).

[5] H. Kino and H. Fukuyama, J. Phys. Soc. Jpn. 652158 (1996).

[6] H. Kondo and T.Moriya, 683170 (1999).

[7] K. Kuroki and H. Aoki, Phys. Rev. B 603060 (1999).

[8] T. Kashima and M.Imada, J. Phys. Soc. Jpn. 703052 (2001).

[9] D.A. Huse and V. Elser, Phys. Rev. Lett. 602531 (1988); B. Bernu et al. Phys. Rev. B 5010048 (1994).

[10] Z. Weihong R.H. McKenzie and R.P. Singh, Phys. Rev. B 5914367 (1999).

[11] N. Read and S. Sachdev, Phys. Rev. Lett.62 (1989) 1694;
O.P. Sushkov et al., Phys. Rev. B63 104420 (2001).

[12] L. Capriotti and S. Sorella, Phys. Rev. Lett.84 3173 (2000).

[13] I. Affleck and Marston, Phys. Rev. B 343774 (1988).

[14] For details, S. Watanabe and M. Imada, unpublished.

[15] A. Kobayashi et al., Chem. Lett. 459 (1987).

[16] T. Komatsu, N. Matsukawa, T. Inoue and G. Saito, J. Phys. Soc. Jpn. 651340 (1996).

[17] A. Fortunelli, and A. Painelli, J. Chem. Phys. 1068051 (1997).

[18] Y. Shimizu, M. Maeda, G. Saito, K. Miyagawa and K. Kanoda, private commun.

[19] J. Schmalian, Phys. Rev. Lett. 814232 (1998).

[20] K. Kuroki et al., cond-mat/0108506. 